\title{
Thyrotropin Receptor: Allosteric Modulators Illuminate Intramolecular Signaling Mechanisms at the Interface of Ecto- and Transmembrane Domain $\$$
}

\author{
Patrick Marcinkowski, Annika Kreuchwig, Sandro Mendieta, Inna Hoyer, Franziska Witte, ${ }^{1}$ \\ Jens Furkert, Claudia Rutz, Dieter Lentz, Gerd Krause, and Ralf Schülein \\ Leibniz-Forschungsinstitut für Molekulare Pharmakologie (FMP), Berlin, Germany (P.M., A.K., S.M., I.H., F.W., J.F., C.R., G.K., \\ R.S.) and Institut für Chemie und Biochemie - Anorganische Chemie, Freie Universität Berlin, Berlin, Germany (D.L.)
}

Received April 29, 2019; accepted July 23, 2019

\begin{abstract}
The large TSH-bound ectodomain of the thyrotropin receptor (TSHR) activates the transmembrane domain (TMD) indirectly via an internal agonist (IA). The ectodomain/TMD interface consists of a converging helix, a Cys-Cys-bridge-linked IA, and extracellular loops (ECL). To investigate the intramolecular course of molecular activation, especially details of the indirect activation, we narrowed down allosteric inhibition sites of negative allosteric modulator (NAM) by mutagenesis, homology modeling, and competition studies with positive allosteric modulator (PAM). From the inhibitory effects of NAM S37a on: 1) chimeras with swapped ectodomain, 2) stepwise $\mathrm{N}$-terminal truncations, 3) distinct constitutively active mutations distributed across the hinge region and ECL, but not across the TMD, we conclude that S37a binds at the ectodomain/TMD interface, between the converging helix, ECL1, and the IA. This is also supported by the noncompetitive inhibition of PAM-C2-activation by S37a in the TSHR-TMD construct lacking the ectodomain. Mutagenesis studies on the IA and ECL were guided by our refined model of the ectodomain/TMD interface and indicate an interaction with the TSHR-specific residues E404 (preceding IA) and $\mathrm{H} 478$ (ECL1). At this new allosteric interaction site, NAM S37a blocks both TSH- and PAM-induced activation of the TSHR. Our refined

models, mutations, and new allosteric binding pocket helped us to gain more detailed insights into the intramolecular course of TSHR activation at the ectodomain/TMD interface, including the delocalization of the converging helix and rearrangement of the conformation of IA. These changes are embedded between the ECL and cooperatively trigger active conformations of TMD.

\section{SIGNIFICANCE STATEMENT}

The intramolecular activation mechanisms of the TSHR appear to be distinct from those of other $\mathrm{G}$ protein-coupled receptors, as the TSHR has a uniquely large $\mathrm{N}$-terminal ectodomain that includes the hormone binding site and an internal agonist sequence. We present new molecular and structural insights into the interface between ectodomain and transmembrane domain in the TSHR, as well as the transfer of activation to the transmembrane domain. This knowledge is critical for understanding activation or inhibition of the receptor by allosteric ligands. We have identified a new allosteric antagonist binding pocket that is located exactly at this interface and possesses specific features that may allow the generation of potent highly TSHR-selective drugs, of potential value for the treatment of Graves' orbitopathy.
\end{abstract}

\section{Introduction}

Together with the lutropin and follitropin receptors, the thyrotropin receptor or thyroid-stimulating hormone (TSH) receptor (TSHR) belongs to a subfamily of glycoprotein hormone

This work was supported by the Deutsche Forschungsgemeinschaft (DFG, German Research Foundation) [Grant number KR1273/4-2].

${ }^{1}$ Current affiliation: Max Delbrück Center for Molecular Medicine (MDC), Berlin, Germany.

https://doi.org/10.1124/mol.119.116947.

S This article has supplemental material available at molpharm. aspetjournals.org. receptors (GPHR) that are the class A G protein-coupled receptors (GPCRs) (Vassart et al., 2004). TSH binds to its receptor and leads to the stimulation of secondary messenger pathways, predominantly involving cAMP (Laurent et al., 1987). Inositol 1,4,5-trisphosphate and diacylglycerol pathways are also activated at higher TSH concentrations (Kero et al., 2007; Song et al., 2010). TSH and the TSHR are key proteins in the control of thyroid function. TSHR is expressed in the thyroid gland but also in retro-orbital fibroblasts. Pathologic activation of the TSHR by autoimmune antibodies that mimic its natural hormone ligand (Rapoport et al., 1998) leads 1) to uncontrolled

ABBREVIATIONS: BSA, bovine serum albumin; CAM, constitutively active mutations; $\mathrm{CH}$, converging helix; DMEM, Dulbecco's modified Eagle's medium; ECD, extracellular domain; ECL, extracellular loops; EDI, $\triangle$ SP-409-764-TSHR; FSH, follicle-stimulating hormone; FSHR, folliclestimulating hormone receptor; GFGQ, truncated TSHR construct TSHR 365-764; GFP, green fluorescent protein; GPCRs, G protein-coupled receptors; GPHR, glycoprotein hormone receptors; HEK, human embryonic kidney; KFLR, truncated TSHR construct TSHR 415-764; KNQK, truncated TSHR construct TSHR 287-764; LRRD, leucine-rich repeat domain; NAM, negative allosteric modulator; PAM, positive and allosteric modulators; PBS, phosphate-buffered saline; PE, R-phycoerythrin; TMD, transmembrane domain; TMH, transmembrane helix; TSH, thyroidstimulating hormone; TSHR, TSH receptor; wt, wild-type. 
production of thyroid hormones by the thyroid gland, causing hyperthyroidism (Graves' disease) and 2) in the eye to exophthalmos (Graves' orbitopathy, GO). Antithyroid drugs available on the market inhibit thyroid hormone synthesis in the thyroid gland but do not act directly on the TSHR and are therefore less effective in the treatment of GO (Sato et al., 2015). Small molecules acting directly on the TSHR are thought to interact allosterically in the transmembrane domain (TMD) as positive and negative allosteric modulators [(PAM, NAM), reviewed in Krause and Marcinkowski (2018)].

The molecular activation mechanisms of TSHR appear to be distinct from that of other GPCRs owing to its uniquely large N-terminal extracellular domain (ECD) in terms of overcoming its inhibitory function (Zhang et al., 1995, 2000; Vlaeminck-Guillem et al., 2002) upon ligand binding (Kleinau et al., 2011). The hormone TSH binds between the two distinguishable receptor parts of the ECD, the leucinerich repeat domain (LRRD) and the hinge region [reviewed in (Krause et al., 2012)]. It is hypothesized that this binding triggers conformational changes at a common convergent center of the LRRD and hinge region that then dissolve the inherent tethered inhibition by the ECD [reviewed in Kleinau et al., 2017)]. A recent peptide screening study identified an internal agonist sequence (TSHR 405-414) that is a highly conserved sequence occurring shortly prior to transmembrane helix (TMH) 1 in GPHR (Brüser et al., 2016). A schematic overview of the nomenclature and topology of TSHR is shown in Fig. 1.

For GPHR, the only structure fragments available are for LRRD with bound stimulating (TSHR 21-260, PDB: 3G04) (Sanders et al., 2007) and blocking (TSHR 22-260, PDB: 2XWT) (Sanders et al., 2011) antibodies and for the folliclestimulating hormone (FSH) bound to LRRD and the hinge region of the follicle stimulating hormone receptor (FSHR)

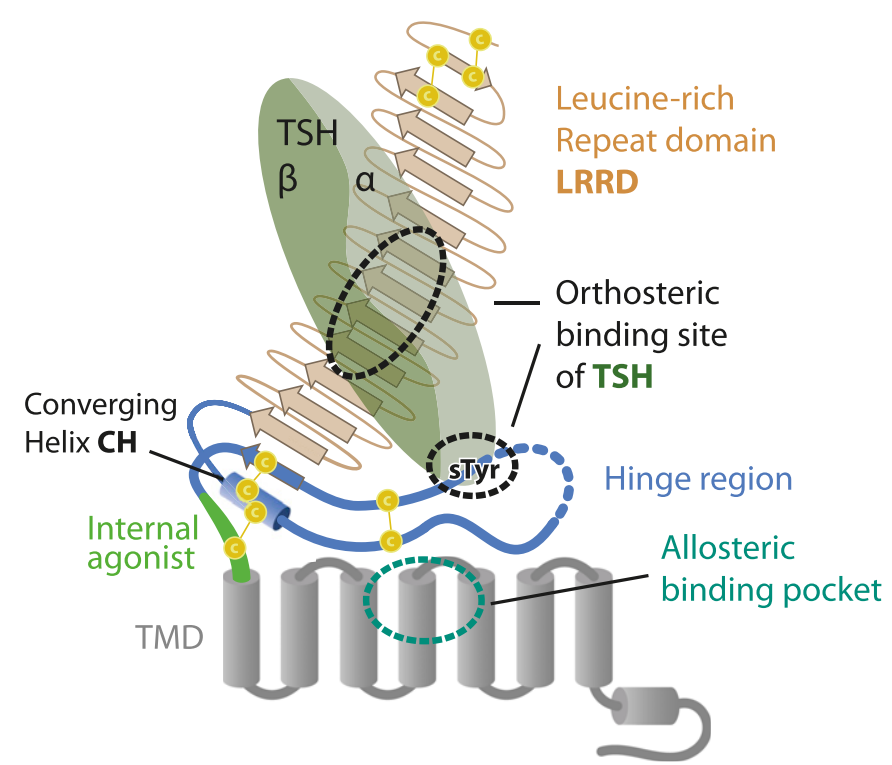

Fig. 1. Outline of the TSH-receptor's nomenclature and topology of the different domains and features. The extracellular orthosteric binding site of TSH is located between the leucine rich repeat domain (LRRD) and the hinge region. The latter contains a converging helix $(\mathrm{CH})$ that uses disulfide bridges to link the LRRD and the internal agonist sequence (green) close to the transmembrane domain (TMD). The TMD contains an allosteric binding pocket.
18-359, PDB 4AY9 (Jiang et al., 2012). Molecular homology models of TSHR have therefore been assembled by variant fragments of the ECD and the transmembrane domain (TMD) using diverse templates (Kleinau et al., 2017).

Single point constitutively activating mutations (CAM) in the transition of LRRD to hinge region on the converging helix (CH) and in the extracellular loops (ECL) of TSHR showed synergistic effects in their combinations as multiple mutations and cooperatively trigger the signal (Kleinau et al., 2008). These and many other CAM [collected in GPHR research resource: www.ssfa-gphr.de (Kreuchwig et al., 2013)] support the hypothesis that the hinge region interacts with the ECL constraining the basal state, which is released/changed upon activation [FSHR reviewed in depth by Briet et al. (2018)].

Nonetheless, due to the lack of the crystal structure of the overall receptor, it is not clear: 1) how ECD and TMD are arranged relative to each other and 2) how the indirect activation of the TMD takes place in detail and 3) whether and how PAM and NAM act on this activation.

On the basis of the mentioned previous findings and by combining mutagenesis, modeling and small ligand modulators, we aim to shed light on these critical points. We have studied the effect of our recently discovered highly TSHR selective small-molecule NAM S37 as racemate and its active enantiomer S37a (Marcinkowski et al., 2019): 1) on stepwise N-terminally truncated TSHR constructs, 2) on the TMD alone and 3) on point mutations distributed across the hinge region, all three ECL and the TMD. The TSHR constructs were activated either by TSH and/or by a smallmolecule PAM-C2 (Neumann et al., 2009, 2016).

\section{Materials and Methods}

\section{Generation of TSHR Mutants}

Unless otherwise specified, all mutants were tagged with green fluorescent protein (GFP) at the intracellular C-terminus to evaluate expression. pTSHR-GFP (wild-type human TSHR cDNA present in the pEGFP-N1 expression vector; Clontech, Heidelberg, Germany) has been described before (Teichmann et al., 2014) and was used as template for the generation of mutants. The sequences of all constructs were verified by Sanger sequencing (Source Bioscience, Berlin, Germany).

Truncated Constructs. Ectodomain truncated TSHR constructs (Fig. 2A) KNQK (287-764-TSHR), GFGQ (365-764-TSHR), and EDI ( $\Delta$ SP-409-764-TSHR) with deleted signal peptide (SP; 1-24-TSHR) were amplified from pTSHR-GFP using standard PCR techniques. To facilitate the deletion of the ectodomain fragments, an EcoRI restriction site was introduced between the sequence encoding TSHR amino acid position C24 and S25 directly after the signal peptide cleavage site. Thereby the amino acids G, I, and Q were added into truncated and wild-type (wt)-TSHR that were believed to have no influence on the structure and function of the receptor constructs.

In EDI, the signal peptide was deleted by exchange with a fragment of the cytomegalovirus promoter from pEGFP-N1 using restriction endonucleases SnaBI and EcoRI. An N-terminal FLAG tag was introduced into truncated and wt-TSHR constructs directly ahead of the EcoRI site by overlap extension PCR (Ho et al., 1989).

Chimeras. For TSHR-FSHR chimeras, the sequences of both receptors were exchanged at the conserved region after leucine-rich repeat 11 (YPSHCCAF), in accordance with the T3 and F3 chimeras of Schaarschmidt et al. (2014) and using restriction-free cloning (van den Ent and Löwe, 2006). They were designated as TSHRxFSHR (TSHRLRRD and FSHR-hinge/TMD) and FSHRxTSHR (FSHR-LRRD and TSHR-hinge/TMD). An N-terminal FLAG tag after the FSHR signal 
A

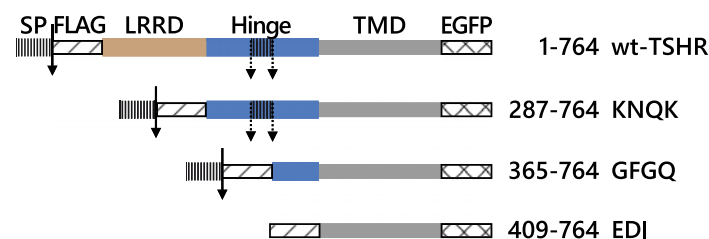

B

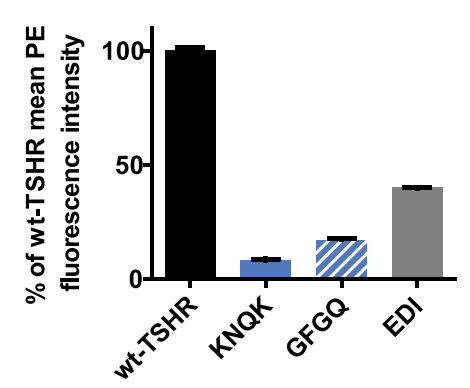

C

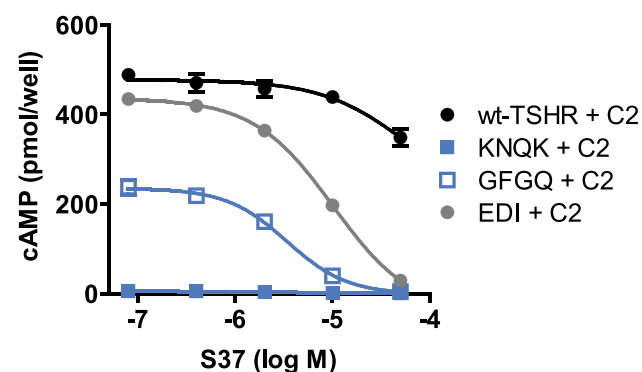

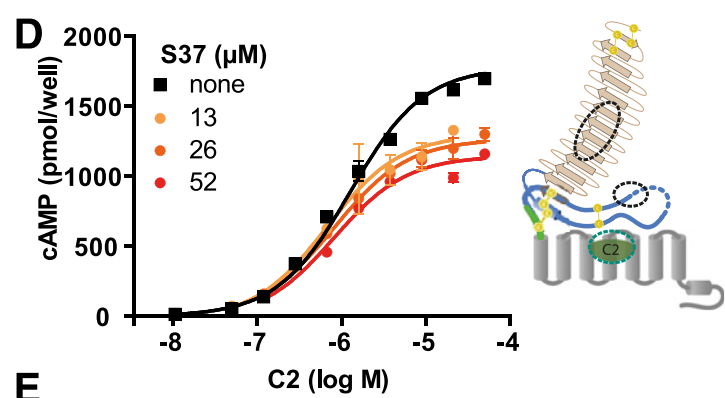

E

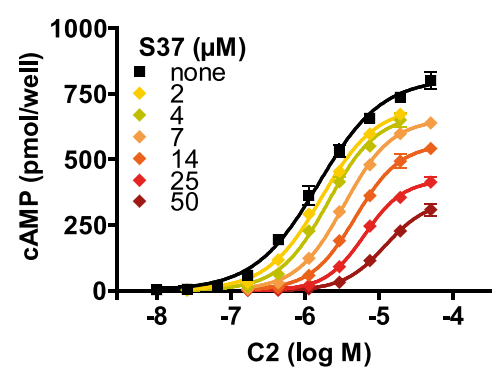

$\mathbf{F}$

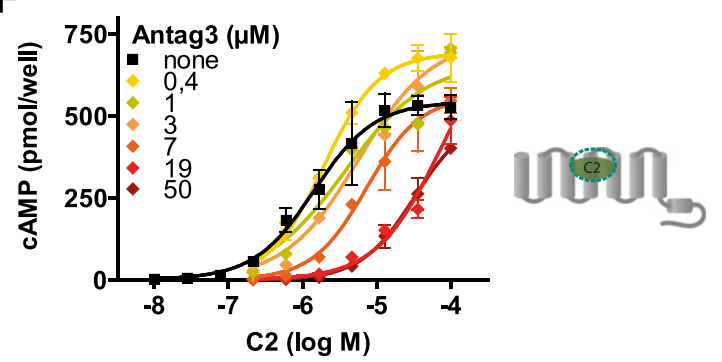

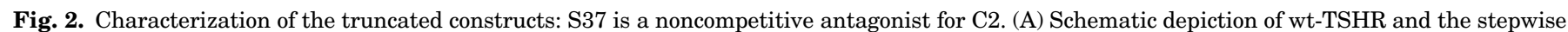

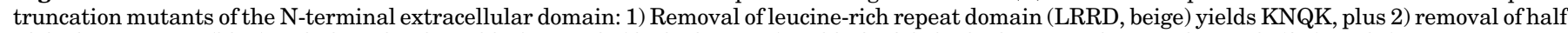

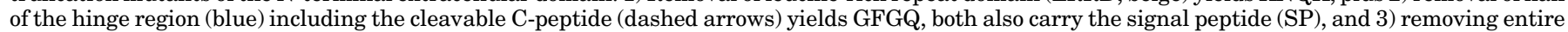

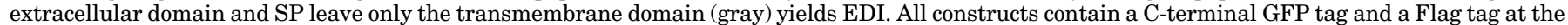

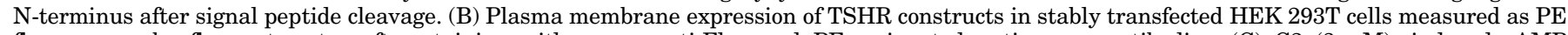

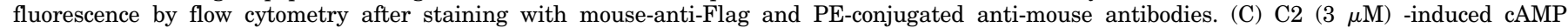

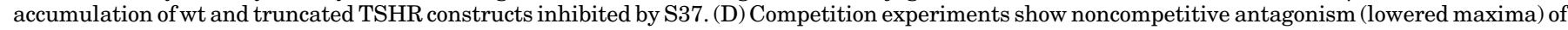

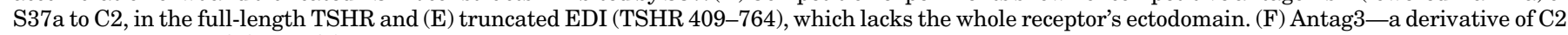
-is a competitive inhibitor of C2 in EDI.

peptide was introduced into FSHR and FSHRxTSHR by overlap extension PCR according to the truncated TSHR constructs. The C-terminally GFP-tagged chimeras were present in the pEGFP-N1 vector. Detailed cloning procedure and primers used will be provided upon request.

Point Mutations. Point mutated hTSHR present in the pcDNA3 expression vector were used from laboratory stock and have been described before (Kleinau et al., 2010). Point-mutated hTSHR present in pEGFP-N1 were generated using site-directed mutagenesis, including the proofreading DNA polymerase PfuTurbo (Agilent).

\section{Cell Culture and Transfection}

Human embryonic kidney (HEK) 293T cells (DSMZ, Braunschweig, Germany) were cultivated in Dulbecco's modified Eagle's medium (DMEM, GlutaMax; Thermo Fisher, Hennigsdorf, Germany) containing $1 \mathrm{~g} / 1$ glucose, $10 \%$ fetal bovine serum (Biochrom, Berlin, Germany), $100 \mathrm{IU} / \mathrm{ml}$ penicillin, and $100 \mu \mathrm{g} / \mathrm{ml}$ streptomycin at $37^{\circ} \mathrm{C}$ in a humidified $5 \% \mathrm{CO}_{2}$ incubator. For transient transfection of HEK $293 \mathrm{~T}$ cells, a mixture of $1 \mu \mathrm{g}$ polyethylenimine and $0.4 \mu \mathrm{g}$ plasmid DNA in serum-free DMEM was added to cells grown in 24 -well plates 1 day after seeding.

For the generation of HEK $293 \mathrm{~T}$ cell lines stably expressing the truncated TSHR, transiently transfected cells were treated with
$400 \mu \mathrm{g} / \mathrm{ml} \mathrm{G} 418$ twice a week. Approximately 4 weeks after transfection, cells were sorted for GFP fluorescence using the BD Aria II cell sorting device (BD biosciences, Erembodegem, Belgium). All cells were routinely tested for mycoplasma infection.

\section{Determination of Cell Surface Expression by Flow Cytometry}

In a 24 -well plate, $2 \times 10^{5}$ cells per well were seeded without selection antibiotics. Three days after seeding, cells were detached with $1 \mathrm{mM}$ EDTA in phosphate-buffered saline (PBS) and blocked for 10 minutes in blocking buffer (PBS, $0.5 \%$ bovine serum albumin, BSA). All steps were performed at $4^{\circ} \mathrm{C}$ on ice. The cells were incubated with primary and secondary antibodies for 30 minutes in blocking buffer. Primary mouse anti-FLAG (clone M2; Sigma) antibody was used diluted 1:1000, and R-phycoerythrin (PE)-conjugated goat anti-mouse IgG secondary antibody (Jackson ImmunoResearch) was diluted 1:50. Cells were washed with blocking buffer three times after each antibody incubation, and 10,000 cells per sample were analyzed using a fluorescence flow cytometer (FACSCalibur; BD biosciences) with a 488-nm argon laser. GFP fluorescence was measured at $510 \pm 20 \mathrm{~nm}$ and PE fluorescence at $585 \pm$ 42-nm bandpass. Each sample was measured in duplicate. The data were analyzed using FCS Express 4 (De Novo Software). Cells were 
gated in a FSC/SSC dot-plot; transfected cells were gated by positive GFP fluorescence compared with nontransfected HEK 293T cells. Plasma membrane receptors were quantified by means of $\mathrm{PE}$ fluorescence using the log Gaussian fitting algorithm in GraphPad Prism 5. Data points represent mean values of duplicates \pm S.D., normalized to wt-TSHR. A single experiment shown represents three independent experiments.

\section{Ligand Treatment and Determination of Intracellular cAMP Accumulation}

In a 24 -well plate coated with poly-L-lysine $(25 \mu \mathrm{g} / \mathrm{ml}$, molecular weight $\geq 300,000$; Sigma), $2 \times 10^{5}$ cells per well were seeded. Stable cell lines were seeded without selection antibiotics and ligand treatment was performed 72 hours after seeding. Transiently transfected cells were treated with ligands 48 hours after transfection. Intracellular cAMP accumulation was measured by radioimmunoassay as described previously (Kleinau et al., 2010). Briefly, cells were washed with $1 \mathrm{ml}$ of stimulation buffer [DMEM GlutaMax supplemented with $10 \mathrm{mM}$ HEPES, 0.5\% BSA, and 0.25 mM 3-isobutyl-1methylxanthine (IBMX)] and incubated for 1 hour at $37^{\circ} \mathrm{C}$ with stimulation buffer alone or stimulation buffer containing bovine TSH (bTSH; Sigma), recombinant human follitropin (rhFSH; R\&D systems) and/or small-molecule ligands at the indicated concentrations. Small molecule TSHR ligands, C2 and Antag3, were a gift from Susanne Neumann and Marvin Gershengorn (National Institutes of Health). The development of S37-rac. and S37a has been described comprehensively (Marcinkowski et al., 2019).

\section{Radioligand Displacement Binding Assay}

The assay was performed using whole-cell membranes prepared from HEK 293T cells stably expressing wild-type human TSHR (HEKTSHR), as described previously (Hoyer, 2014). For each sample, a membrane preparation containing $10 \mu \mathrm{g}$ total protein and 30,000 cpm ${ }^{125}$ I-bTSH (TRAK kit, B.R.A.H.M.S; Thermo Fisher Scientific) were incubated with increasing concentrations of cold ligands in a final volume of $200 \mu \mathrm{l}$ in binding buffer $(50 \mathrm{mM}$ Tris, $2 \mathrm{mM}$ EGTA, $10 \mathrm{mM} \mathrm{MgCl} 2,0.5 \mathrm{~g} / \mathrm{l} \mathrm{BSA}, 213 \mu \mathrm{g} / \mathrm{ml}$ bacitracin, $80 \mu \mathrm{g} / \mathrm{ml}$ benzamidine, $17 \mu \mathrm{g} / \mathrm{ml}$ aprotinin, $3 \mu \mathrm{g} / \mathrm{ml}$ soy bean trypsin inhibitor, $0.5 \mathrm{mM}$ phenylmethyl sulfonyl fluoride, $\mathrm{pH} 7.5$ ) for 2 hours at $25^{\circ} \mathrm{C}$. Membranes were harvested on GF/C glass fiber filters (IH-201-C; Inotech) and washed five times with cold PBS. Radioactivity of bound ligand was then measured in a gamma-counter.

\section{Data Analysis}

The present work was exploratory and did not test a statistical null hypothesis. The individual, independent experiments for cAMP accumulation and radioligand binding were performed in triplicates and for concentration-response curves in duplicates. If not stated otherwise, raw data are shown from a single experiment representative of three independent experiments, and normalized data are shown as average of three independent experiments. Data were analyzed using the software GraphPad Prism 5 and are shown as mean and S.D. For concentration-dependent curves x-axis values were log-transformed and $y$-axis mean values were fitted using a threeparametric (bottom, top, $\mathrm{E} / \mathrm{IC}_{50}$ ) sigmoidal curve.

\section{Crystal Structure Determination of S37a}

The racemate $\mathrm{S} 37$ had been separated into its enantiomers S37a (eluted first) and S37b (eluted second) by chiral high-performance liquid chromatography, as described previously (Marcinkowski et al., 2019).

Crystals could be obtained from a super-saturated solution of S37a in 1,4-dioxane. Diffraction data were collected on a Bruker-AXS D8 Venture instrument equipped with an Incoatec Microfocus Source using $\mathrm{Cu} \mathrm{K} \alpha$ radiation and a Photon detector. The APEX3 software
(Bruker AXS Inc., 2016) was used for data collection and reduction. The structure was solved and refined using SHELXT (Sheldrick, 2015b) and SHELXL (Sheldrick, 2015a), respectively. The absolute configuration of 37a was unequivocally determined by an X-ray crystal structure analysis by anomalous dispersion with a Flack parameter of 0.037(4). ORTEP for Windows (Farrugia, 1997) was used to create the drawing of the structure.

\section{Homology Modeling}

Generation of the TMD model of TSHR in the inactive state began with assembly of the best transmembrane helix templates on the basis of our published fragment-based molecular modeling approach [GPCR-Sequence-Structure-Feature-Extractor (GPCR-SSFE) (Worth et al., 2017)]. The loops were generated with the help of the GPCR-ITASSER web resource (Zhang et al., 2015).

To generate full-length models, we updated the previously generated ECD/TSH complex model (Kleinau et al., 2017) the basis of which is the FSHR/FSH crystal structures [4AY9, 4MQW, (Jiang et al., 2012)]. The ECD model also contains the hinge region, particularly the short $\mathrm{CH}$, and part of the internal agonist. We truncated the last residues $\mathrm{P} 407$ and $\mathrm{C} 408$ of the ECD so that $\mathrm{C} 284$ on $\mathrm{CH}$ would be accessible. At the TSHR model of the TMD (inactive state), we added a part of the internal agonist ${ }_{408}$ CEDIMGY prior to TMH1, using as template the crystal structure of a homologous sequence fragment CENVIGY (PDB: 1DQA). The resulting extended TMD construct then contained a freely accessible C408. For docking the ECD to the TMD, two web tools were used, HADDOCK (van Zundert et al., 2016) and ITASSER (Zhang et al., 2015), exploiting the user-specified restraint (inter-residue or distance restraints) of the existing disulfide bond between $\mathrm{C} 284$ at $\mathrm{CH}$ of the ECD with $\mathrm{C} 408$ then being located in the TMD model. Both approaches generated a variety of docking clusters. From the best scoring clusters, we chose for further consideration the one that was predicted in an identical configuration by the two methods.

The crystal structure of S37a (Fig. 5) was docked into the TMD model (corresponding to the EDI construct) of the inactive state using the docking module Glide of the Maestro11 software (2017; Schrödinger, LLC, New York, NY). Glide docking methodologies use hierarchical filters allowing flexible ligand positioning in the receptor binding-site region. As a first step, the model quality was checked by the Protein Preparation Wizard. Subsequently, a grid defining the shape and properties of the binding site region was set up, on the basis of the previously published characterization of the binding site (Hoyer et al., 2013) of the TSHR TMD. During the docking process, exhaustive ligand torsion sampling and refinement of selected docking poses led to the selection of high affinity, low Glide scoring poses of S37a. Finally, the selected poses were minimized with full ligand flexibility in a postdocking minimization step.

\section{Results}

To improve our understanding of the intramolecular course of molecular activation across the entire TSHR, especially details of the indirect activation of the TMD and how this is influenced by NAM, we narrowed down the potential target sites of NAM.

\section{Truncated TSHR Constructs}

First, three truncated TSHR constructs related to previous reports (Vlaeminck-Guillem et al., 2002) were generated. They were shortened stepwise by parts of the ECD but retain the TMD. The first truncation, TSHR 287-764 (starting with KNQK), lacked the LRRD but still also contained the entire extracellular hinge region. The second truncation, TSHR 365764 (GFGQ), contained only the second half of the hinge region 
after the C-peptide, including the internal agonist. The shortest construct, TSHR 409-764 (EDI), consisted only of the TMD. In contrast to construct 415-764 [called KFLR in VlaeminckGuillem et al. (2002)], our EDI also contained six preceding amino acids to constitute the complete transmembrane helix 1 (TMH1) (Fig. 2A).

Since the N-terminally truncated TSHR-constructs cannot be activated by TSH (Vlaeminck-Guillem et al., 2002), the activation with the small-molecule agonist called C2 was a prerequisite for antagonist treatment. The truncations were activated by $\mathrm{C} 2$ with different efficacies in transiently transfected HEK 293T cells (Supplemental Fig. 1). The $\mathrm{EC}_{50}$ of $\mathrm{C} 2$-induced cAMP production was $2 \mu \mathrm{M}$ in KNQK, but $1 \mu \mathrm{M}$ in wt-TSHR and in the other truncated constructs. This demonstrates the mutant's functionality in terms of $\mathrm{G}_{\mathrm{s}}$ activation, which has also been previously shown for the TSHR truncation KFLR (Neumann et al., 2009).

Second, stable HEK 293T cell lines expressing the constructs were generated. Their cell surface expression was 8\%-40\% of wt-TSHR (Supplemental Fig. 2). Constitutive activity for the truncated constructs has been described for analogous constructs (Vlaeminck-Guillem et al., 2002), which we generally confirmed (Supplemental Fig. 3A).

Figure 2D shows that all truncated constructs were inhibited by S37, which proves in the first place that it binds to the TMD of TSHR. Moreover, binding to the LRRD was excluded for the active enantiomer S37a by ECD/TMD swapping TSHR-FSHR chimeras (Supplemental Fig. 4). The effects of respective hormones on such chimeras have been described previously (Schaarschmidt et al., 2014). S37 and S37a are selective for TSHR and do not inhibit the FSHR. Therefore they should inhibit only the chimera containing the TSHR TMD, as was the case for S37a.

Interestingly, compound S37 had a very different effect in the truncated TSHR than in wt-TSHR. In wt-TSHR, C2 activation was inhibited by $25 \%$ when $50 \mu \mathrm{M}$ S37 was used (Fig. $2 \mathrm{C}, \mathrm{IC}_{50}>50 \mu \mathrm{M}$ ). However in the truncated constructs C2-induced cAMP signaling was completely inhibited at $50 \mu \mathrm{M}$ and the $\mathrm{IC}_{50}$ was $3 \mu \mathrm{M}$ for the KNQK and GFGQ and $10 \mu \mathrm{M}$ for the EDI construct (Fig. 2C, blue and gray curves, respectively).

Although the TSHR ECD is dispensable for S37 binding (activation of EDI is inhibited by S37), the ECD seems to have a strong influence on the function of S37 (Fig. 2C), especially in contrast to $\mathrm{C} 2$, whose $\mathrm{EC}_{50}$ is only changed slightly upon removal of the ECD (Supplemental Fig. 1).
In previous studies, cAMP measurements using full length wt-TSHR indicated that S37 acts as a competitive antagonist for TSH (Marcinkowski et al., 2019). To prove that S37a does not actually displace bTSH, we performed a radioligand binding assay. As expected, we were able to show that S37a does not inhibit ${ }^{125}$ I-bTSH binding to TSHR (Fig. 3).

Moreover, S37 showed noncompetitive antagonism to agonist $\mathrm{C} 2$ in the cAMP assay for the full-length TSHR (Fig. 2D), which was confirmed for the EDI construct that lacks the entire ECD (Fig. 2E), demonstrating that S37 binds to the TMD but not at the same binding site as C2. To prove the validity of the competition assay, we repeated it in the EDI construct with the inverse agonist Antag3 (Neumann et al., 2014), which is a derivative of C2 and therefore is supposed to inhibit activation by $\mathrm{C} 2$ competitively. Indeed, in contrast to S37, we obtained right-shifted concentration-response curves of $\mathrm{C} 2$ when the Antag3 concentration was increased (Fig. 2F), indicating competitive antagonism and, hence, overlapping binding sites for C2 and Antag3.

These results clearly demonstrate that the binding site for S37 must be located at the TSHR-TMD but is different from that of the known allosteric $\mathrm{C} 2$ binding site in the TMD.

\section{Effects of S37a on TSHR Constitutively Activating Mutants}

Since S37 and S37a bind to the TMD but not in the classic pocket like $\mathrm{C} 2$, we further considered potential interaction sites of S37a between the extracellular vestibule on the top of the 7TM bundle and the ECD. Therefore we tested the inhibitory effect of S37a on known constitutively activating mutants of TSHR [selected from www. SSFA-gphr.de (Kreuchwig et al., 2013)] located on $\mathrm{CH}$ of the hinge region (S281Q), internal agonist (N406D), ECL1 (I486F), ECL2 (I568T), and ECL3 (V656F). CAM in the hinge region and ECL of TSHR probably change particular interactions between ECD and TMD. CAM on variant positions across the TMD (V421I, Y466A, T574A, D619A, M637W, Y643F, and $\mathrm{L} 645 \mathrm{~V}$ ) of TSHR are also thought to track other potential binding sites on TMD. CAM in the TMD indicate positions/residues that are important for stabilizing the basal receptor conformation in the wild-type receptor and are potential switches for receptor activation (Kleinau et al., 2010, 2017; Hoyer et al., 2013). Therefore, different inhibitory effects of S37a depend on particular CAM locations and should contribute to understanding the molecular course of activation and delineation of the binding site.
A

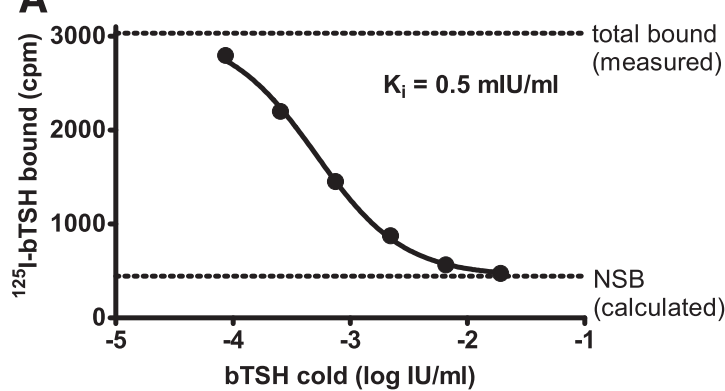

B

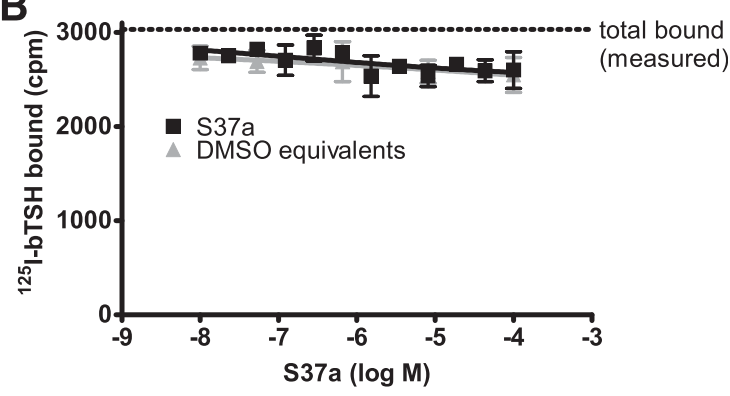

Fig. 3. Radioligand binding study reveals that S37a does not inhibit bTSH binding to TSHR up to a $100 \mu \mathrm{M}$ concentration. ${ }^{125} \mathrm{I}$-bTSH (constant 30,000 $\mathrm{cpm}$ ) and increasing concentrations of nonlabeled bTSH (A) or S37a (B) were incubated with HEK-TSHR membranes. 

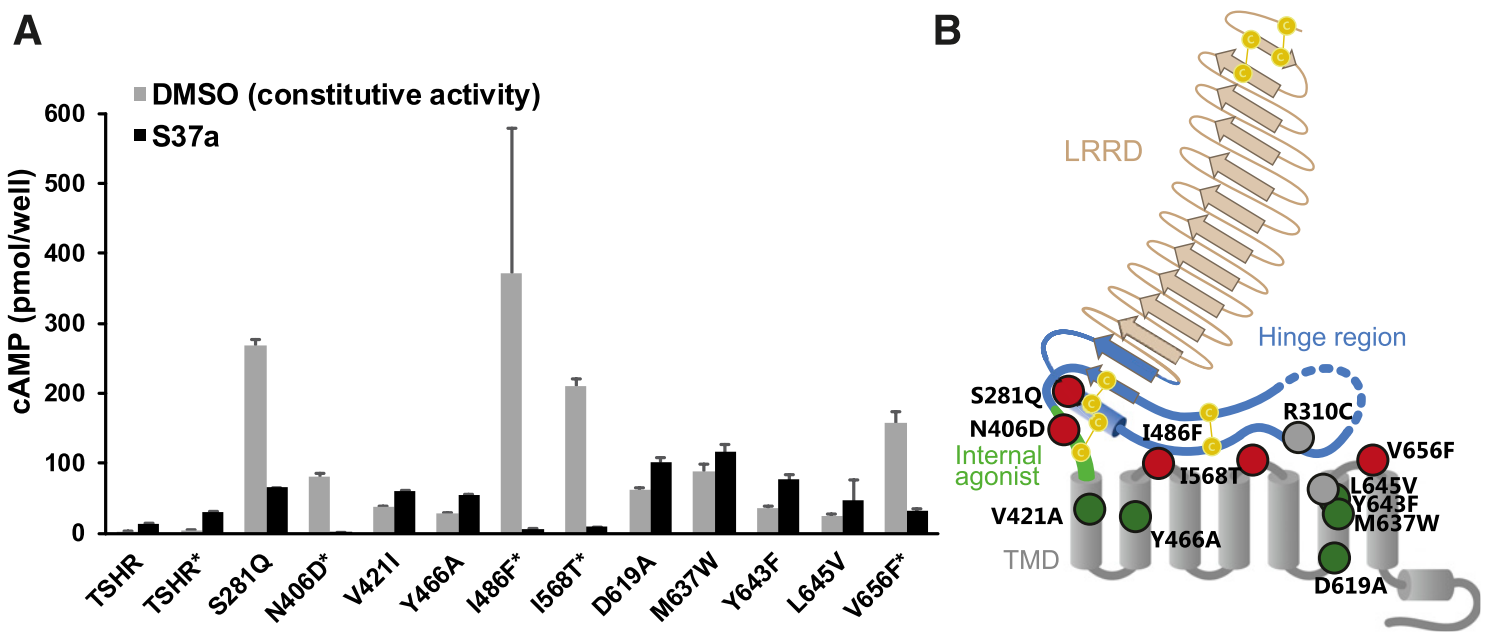

TSHR variant

Fig. 4. Constitutively activating mutations (CAM) of the TSHR are either inhibited by S37a, not affected, or further activated, depending on their location. (A) Transiently transfected HEK 293T cells were treated with $100 \mu \mathrm{M}$ S37a (black) or the equivalent amount of DMSO (gray). *Indicates Flag-receptor-GFP. All the other receptors are untagged. Columns show mean values of cAMP formation of a single experiment performed in triplicates \pm S.D. It is representative of two independent experiments. (B) Scheme of TSHR indicating the locations of CAM that are inhibited by S37a. These are located at the hinge or at the extracellular loops (red). CAM that are not affected (gray) or activated (green) by S37a are located at TMH.

It is striking that S37a clearly inhibits highly elevated cAMP production (gray/black, Fig. 4A) of those CAM of TSHR that are located in: 1) the converging helix, 2) the internal agonist of the hinge region, and 3) the ECL (red in Fig. 4B). This suggests that S37a blocks conformational changes of activation in these particular regions located at the interface between ECD and TMD.

In contrast, those CAM distributed across the TMD cause moderate or slightly elevated cAMP and could not be inhibited by S37a (Fig. 4A; green 4B). The observed slight partial agonism of S37a at wt-TSHR is more or less retained, suggesting that the compound does not, or only to a minor extent, influence CAMs located on the transmembrane helices.

These observations suggest that the site of action of S37a is more probably harbored at the interface between the hinge region and ECL than in the known GPCR ligand binding pockets between the helices.

\section{Docking of S37a Crystal Structure into Model of the TSHR}

The crystal structure of the enantiopure compound S37a containing seven chiral centers was determined by X-ray crystal structure analysis, resulting in a bent structure (Fig. 5) that confirmed our previously predicted absolute configuration (4aS,5S,5aR,8aR,9R,9aS,10R)-7,10-diphenyl5,5a,8a,9,9a,10-hexahydro-5,9-methanothiazolo $\quad\left[5^{\prime}, 4^{\prime}: 5,6\right]$ thiopyrano [2,3-f]isoindole-2,6,8(3H,4aH,7H)-trione (Marcinkowski et al., 2019).

Although the TMD model of TSHR construct EDI lacks the entire ECD in the inactive state, NAM S37a was docked into it because truncation mutations demonstrated the inhibitory interaction of S37a even in the TMD alone. Since the $\mathrm{N}$-terminal residue ${ }_{409}$ EDIMGY is part of the internal agonist, we used for it a homologous sequence fragment from the crystal structure (PDB 1DQA) as corresponding template prior to TM1. In the truncated EDI construct, the largely accessible extracellular vestibule between TMH1, 2, 3, and 7 was constricted by residues EDIMGY, where E409 and D410 in our model are located along ECL3 in the vicinity to Y643
(TMH6) and K660 (TMH7) respectively. The residues I411, M412 are embedded in the extracellular vestibule by hydrophobic residues on TMH7, TMH1, TMH2, and ECL2 (I568).

Our highest scored docking pose of S37a into the binding cavity of the truncated EDI construct was covered by the internal agonist (fragment), TMH2 (H478), ECL1 (I486), and ECL2 (Fig. 6). This is supported by the suppressive effects on particular CAM (Fig. 4), whose positions I486 in ECL1 and partly I568 in ECL2 spatially cover the binding site of S37a (indicated by an asterisk in Fig. 6).

The binding site between TMH1, 2, 3 and the internal agonist (Fig. 6) does not overlap with the allosteric binding

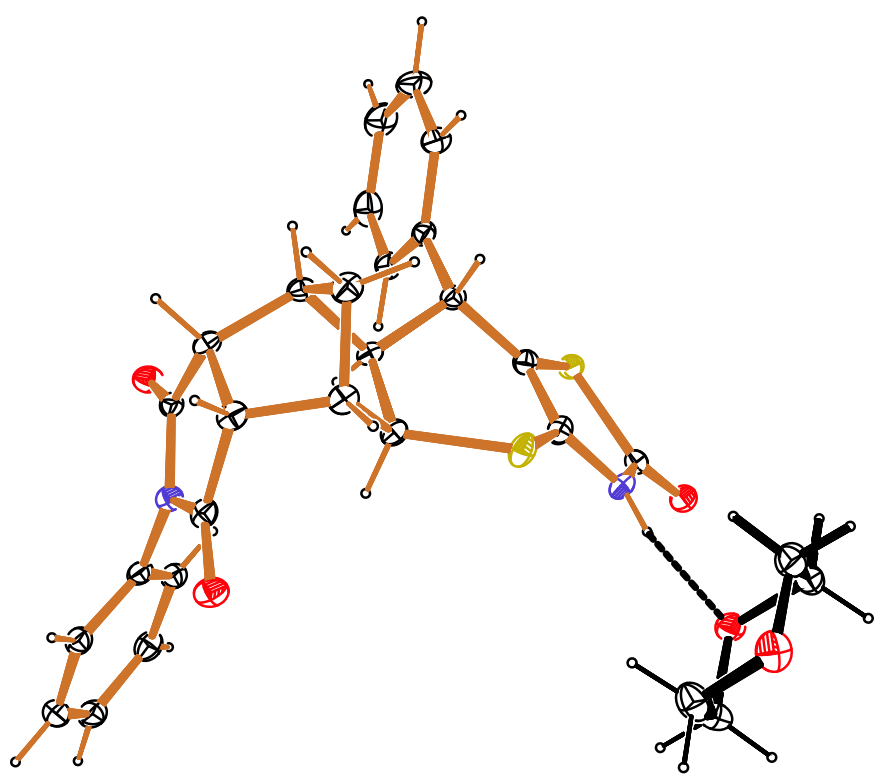

Fig. 5. Crystal structure of S37a (orange) in complex with solute 1,4-dioxane (black). The image was generated using the software Ortep3 for Windows v2014.1 [University of Glasgow, (Farrugia, 2012)]. Crystallization data were deposited in the Cambridge Crystallographic Data Centre under the CCDC number 1894120. 
pocket of C2 (dark blue, Fig. 6), which is consistent with the noncompetitive inhibitory effect of S37a on the truncated TSHR constructs.

As template for the ectodomain model of TSH/TSHR, the FSH-bound fragment of the FSHR ectodomain crystal structure (PDB: 4MQW_B) was used. At its C-terminal end, this contained the $\mathrm{CH}$ and part of the internal agonist, which are linked by the conserved disulfide bridge (C283-C408).

For the refined ECD/TMD interface, the full-length TSHR inactive state model (Fig. 7A) shows that $\mathrm{CH}$ interacts with ECL1 and that the residues S281 (CH) and I486 (ECL1) are therefore spatially very close to each other. $\mathrm{CH}$ is covalently linked via a disulfide bridge to the internal agonist embedded between ECL2 and ECL3 (Fig. 7B). Moreover, the full-length TSHR model indicates that S37a is therefore immersed in a pocket similar to that in the model of the EDI-TSHR construct. However, in this case S37a interacts additionally with the $\mathrm{CH}$ (S281* CAM), E404, and residues of the internal agonist (F405, N406*) (Fig. 7C), which are missing in the EDITSHR model. TSHR positions S281* (CH), N406* (internal agonist), I486*(ECL1), I568* (ECL2), V656* (ECL3), whose $\mathrm{CAM}^{*}$ (visualized as spheres in Fig. 7B) are in close vicinity to S37a, can be suppressed by S37a (Fig. 4).

\section{Effects of S37a on Selected Mutants near ECL and Internal Agonist}

The binding site model was used for the selection of additional site-directed mutations.

Seven different point-mutated TSHR variants (Fig. 8B) were generated that were located in close proximity to one of the predicted S37a docking poses, and cAMP signaling was investigated. All mutants could be activated by bTSH (Supplemental Fig. 5) and were subsequently treated with

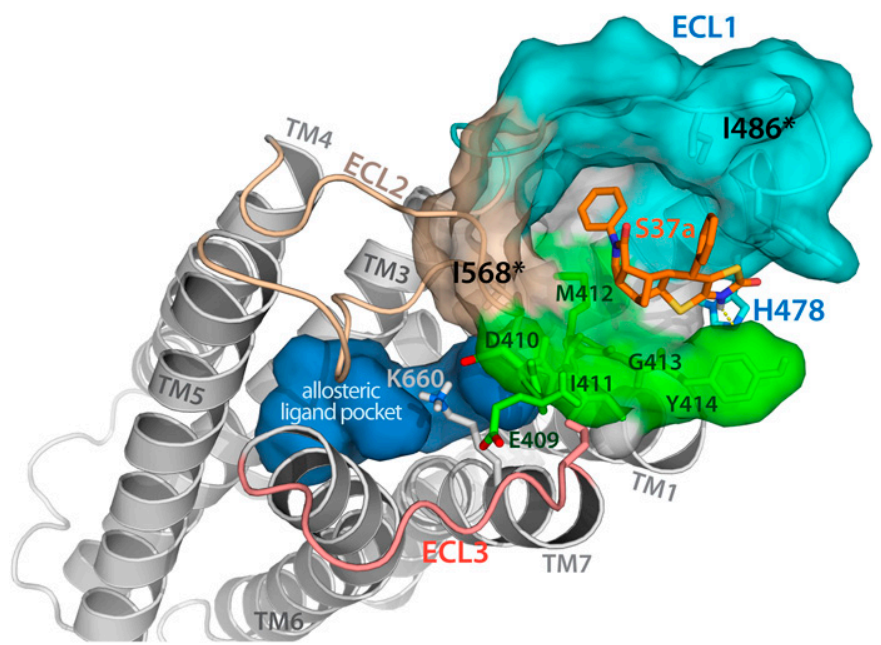

Fig. 6. Docking studies into homology model of the EDI -TSHR construct suggests a binding cavity for the NAM S37a (orange) in the extracellular vestibule among the extracellular loops. This S37a cavity is situated distantly from the binding site of small-molecule agonist $\mathrm{C} 2$. In contrast to $\mathrm{S} 37 \mathrm{a}$, the allosteric binding pocket of $\mathrm{C} 2$ is situated deeper in the TMD (dark blue), where the orthosteric ligand binding pocket is located in many other GPCRs of the rhodopsin family. The fragment ${ }_{409}$ EDIMGY $_{414}$ of the internal agonist (green) is embedded between ECL3/TMH7 (E409, D410), TMH1/TMH7 (I411), TMH2 (M412), and ECL1 (cyan). $\mathrm{S} 37 \mathrm{a}$ is immersed between ECL1, ECL2 (wheaten color) and the fragment of the internal agonist. It interacts with $\mathrm{H} 478$ and is located between positions I486* (ECL1) and I568* (ECL2) whose CAM (*, see Fig. 3) are strongly inhibited by S37a.
S37a. Figure 8A clearly shows that the two mutants E404A and H478A were not inhibited by S37a, whereas in Y414A, Y414F, E480A, and S657A the antagonistic effect was about $60 \%$ at $100 \mu \mathrm{M}$ S37a, which is similar to wt-TSHR. In S567A, the compound showed only $36 \%$ inhibition of cAMP accumulation at $100 \mu \mathrm{M}$. These results indicate that the two TSHspecific residues E404 and H478 and possibly S567 are critical contact points for S37a.

\section{Discussion}

To reveal molecular details of how activation is conveyed at the ECD/TMD interface, we used wt-TSHR and truncated TSHR constructs to investigate details of the indirect and direct activation mechanism of the TMD by TSH, CAM, and PAM C2 and studied how this is blocked by the negative allosteric modulator NAM S37a.

In a previous study, Schild plot analyses of TSHR signaling indicated that S37 is a competitive antagonist for TSH stimulation of cAMP. On the other hand, NAM S37a appeared to be a noncompetitive antagonist of $\beta$-arrestin 1 recruitment, which suggested that S37a may bind at the TSHR ECD (Marcinkowski et al., 2019).

\section{Narrowing Down the Binding Site of S37a}

In reviewing this assumption, we were able to prove by LRRD and hinge/TMD swapping of TSHR/FSHR chimeras and stepwise N-terminal truncations that the LRRD and hinge region of TSHR are dispensable for S37a binding. In addition, a radioligand binding study proved that ${ }^{125} \mathrm{I}-\mathrm{bTSH}$ could not be displaced by S37a (Fig. 3B). Instead, the previously observed competition of S37 and TSH (Marcinkowski et al., 2019) must have been an indirect effect, probably elicited by interaction of S37 with determinants of the TSHR hinge region.

It has been shown by mutagenesis that PAM C2 binds allosterically at TSHR, inside the TM bundle (Neumann et al., 2009). Its potential binding pocket between TMH3, 5, and 6 (Neumann et al., 2016) is equivalent to the ancestral orthosteric ligand binding site of many GPCRs (Wacker et al., 2017). As NAM S37 noncompetitively inhibits activation by $\mathrm{C} 2$, one can conclude that S37a binds elsewhere and does not bind into this particular pocket in the TMD. As inhibition with S37 was also possible in the truncated TSHR containing only the TMD, the presence of a second allosteric binding site within the TMD was conceivable. Moreover, we show here that wild-type and truncated TSHR constructs are activated by $\mathrm{C} 2$ with similar $\mathrm{EC}_{50}$ (Supplemental Fig. 1), which implies that $\mathrm{C} 2$ activates the receptor without involvement of the TSHR-ECD, as its absence does not change the affinity of C2.

We assumed that S37a might bind to a noncanonical receptor site similar to one of those that have been recently discovered for ligands on other GPCRs, for example, at an intracellular site or at the interface between TMH and membrane [reviewed in Wacker et al. (2017)]. Therefore the inhibitory effect of S37a on CAM was investigated not only on positions in the hinge region but also on positions distributed across the entire TMD, including intracellular sites. In this context, it should be noted that CAM may not only have direct effects via the mutant residue but may also have indirect effects on conformations elsewhere in the receptor. Therefore, 


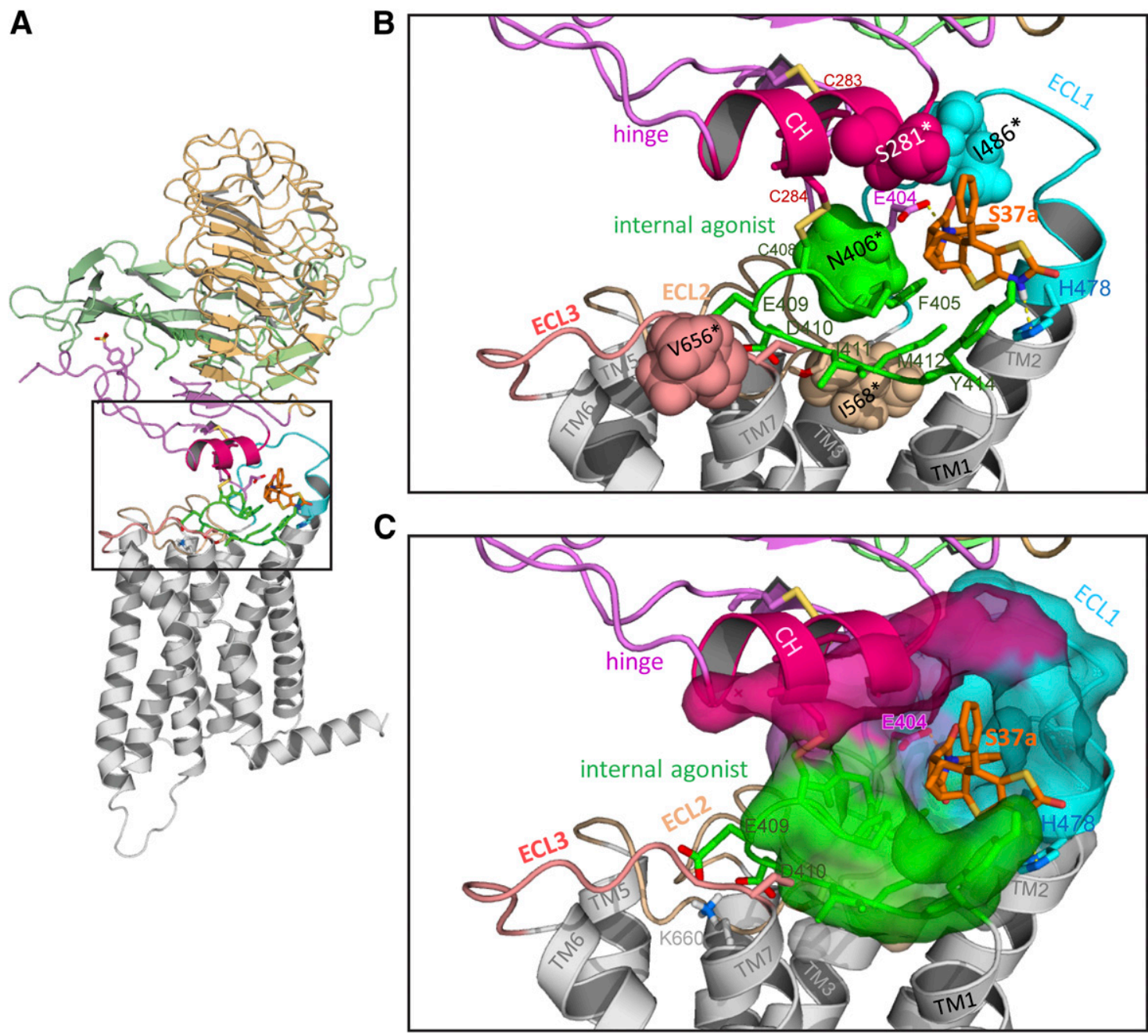

Fig. 7. Refined full-length homology model of the TSHR locked in the inactive state by NAM S37a. (A) TSH (pale green) bound between LRRD (beige) and hinge (magenta) with docked NAM S37a (orange) in an allosteric binding site at the newly modeled ECD/TMD interface (boxed). (B) The converging helix (dark pink) is linked via disulfide bridges with the hinge and the internal agonist (F405-Y414 green). CH interacts with ECL1 (cyan). The internal agonist is placed between ECL1/ECL2 (F405), ECL2/TMH7-ECL3 (E409, D410), as well as between TMH1 (I411) and TMH2-ECL1 (M412). TSHR positions S281* (CH), I486 *(ECL1), I568* (ECL2 wheaten), V656* (ECL3, salmon), whose CAM* (visualized as spheres) can be suppressed by S37a (Fig. 3), are in close proximity to S37a (orange), which (C) is immersed in a pocket and is also bound by the TSHR-specific residues E404 and H478.

we have differentiated constitutive mutations only between those in which S37a inhibits or does not inhibit (see Fig. 4). It is interesting that this differentiation also discriminates between extracellular and transmembrane mutant residues.

S37a suppressed elevated cAMP of CAM positions located in the hinge and the extracellular loops only but had no such effects on CAM positions located in the remaining TMD. Therefore S37a seems to interact at the interface of ECD and TMD rather than on intracellular or membrane interfacial sites.

\section{Verifying New Allosteric Binding Site for NAM S37a at the Interface between ECD and TMD}

Our homology model of the TSHR TMD suggests a binding site for the NAM S37a among the extracellular loops in the vestibule between TMH 1, 2, 3 and the internal agonist (Fig. 6). This is distant from the binding site of PAM C2, which is located deeper in the TMD in between TMH 3, 5, 6 (Neumann et al., 2016). The binding site is consistent with the inhibitory effect of S37 on the different truncated constructs. Any uncertainties about the absolute configuration of the active enantiomer S37a could be cleared up by X-ray crystallography of the compound that was used for docking.

However, the NAM S37a occupies a hitherto unknown allosteric pocket at the ECD/TMD interface that is not related to the established allosteric binding pocket of TSHR nor to the corresponding common orthosteric binding pocket of other GPCRs of the rhodopsin family [reviewed in Wacker et al. (2017)].

The possibility that a NAM could bind in the ECD/TMD interface even near the internal agonist can also be assumed from the fact that the internal agonist as isolated peptide FNPCEDIMGY activates the GPHR, albeit at very high concentrations (Brüser et al., 2016).

Our refined full-length TSHR model substantiates the existence of a binding pocket for NAM S37a at the ECD/ TMD interface, where S37a interacts with E404 (prior internal agonist) and H478 (TMH2). Their substitution with alanine abrogates the antagonism of S37a, which indicates loss of the compound's affinity at these points or in close proximity. Moreover, this is strongly supported by the facts 
A

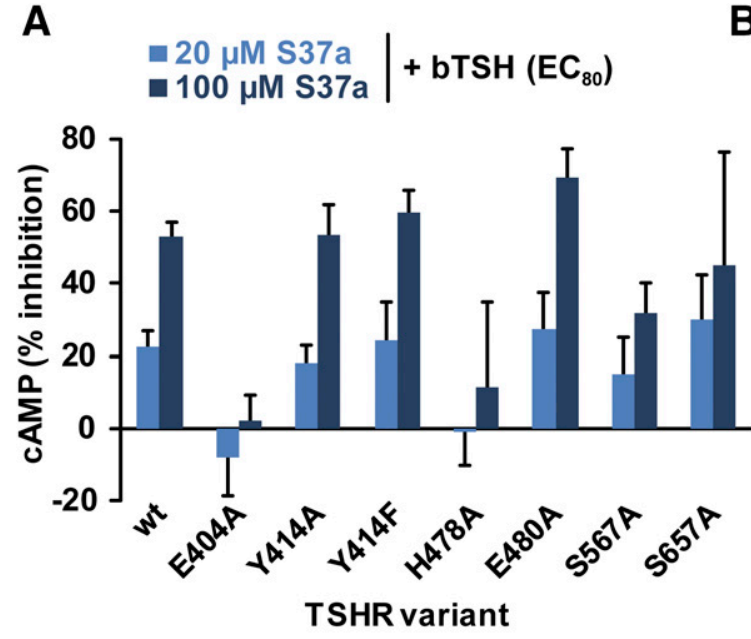

B

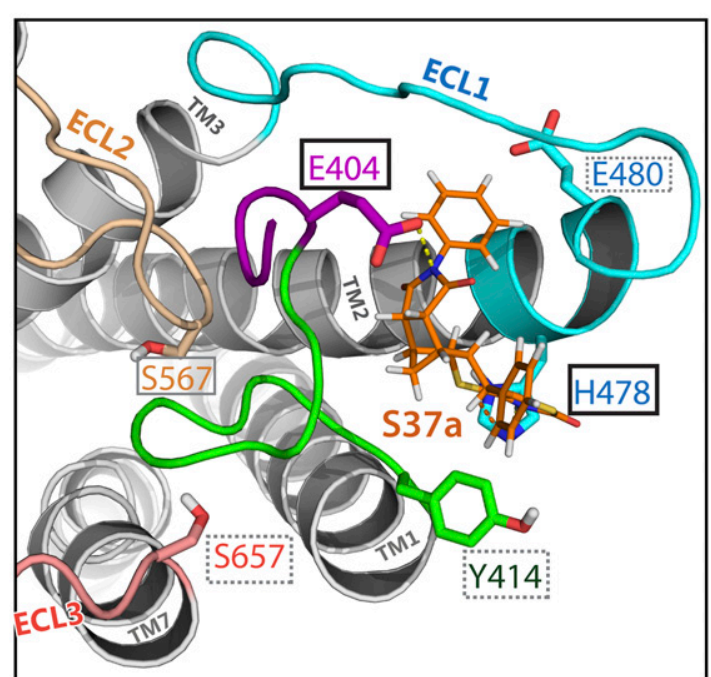

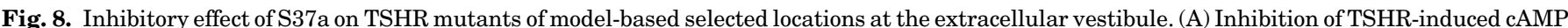

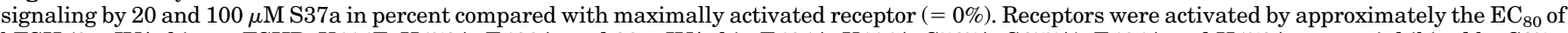

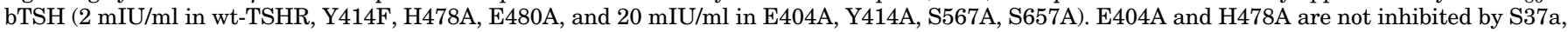

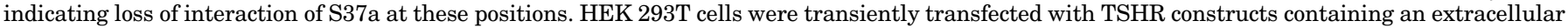

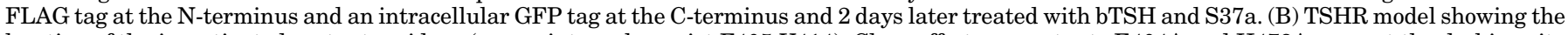

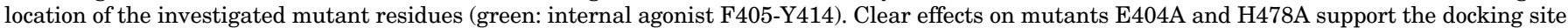
and TSHR selectivity of S37a (orange).

that S37a is highly TSHR-selective (Marcinkowski et al., 2019) and that residues E404 and H478 are both TSHRspecific (see http://www.ssfa-gphr.de/alignment.php).

Other previous experimental findings support the modeled binding site of S37a. The aromatic rings of S37a are surrounded by aromatic residues Y279 $(\mathrm{CH}), \mathrm{F} 405$ (internal agonist), and Y481 (TMH2/ECL1), which were demonstrated as essential for TSHR functionality (Jaeschke et al., 2006; Mueller et al., 2006). This is also valid for residue I486 on ECL1 (Fig. 7B), which can be constitutively activated by mutations (Kleinau et al., 2008).

\section{Course of Intramolecular Activation Mechanism at the ECD/TMD Interface}

Homology models of the entire TSHR and mutation data suggest an important role for the converging helix $(\mathrm{CH}$, 280-288) when it acts as a pivot of the hinge region during the molecular activation mechanism. The $\mathrm{CH}$ is fastened via disulfide bridges (Ho et al., 2001, 2008) [for LHR (Bruysters et al., 2008)] on one side (Cys283-C398) to the additional 13th beta strand that extends the beta sheet of the LRRD and on the other side (C284-C408) to the internal agonist [405-414, (Brüser et al., 2016)].

$\mathrm{CH}$ and the internal agonist sequences are both embedded in between the ECLs of the seven TMH [reviewed in Kleinau et al. (2017) and Krause and Marcinkowski (2018)]. According to our own and other molecular models of TSHR (Kleinau and Vassart, 2017), CH interacts with ECL1, as is also supported by the strong CAM of S281Q (located at $\mathrm{CH}$ ) and $\mathrm{I} 486 \mathrm{~F}$ (located at ECL1) (Fig. 7B). It has been suggested previously that the functionally significant Ser281 interacts with the ECL1 (Jaeschke et al., 2006), as is supported by crosslinking studies (Schaarschmidt et al., 2016). Our NAM S37a is also able to abrogate the CAM N406D (internal agonist), I568T (ECL2), and V656F (ECL3). This reflects the cooperativeness of the three ECL, as previously described (Kleinau et al.,
2008), and now additionally illustrates their interrelationships to the $\mathrm{CH}$ and the internal agonist. According to our refined TSHR model, the complete internal agonistic sequence is arranged between all three loops and is even embedded between the outermost parts of TMH 1, 2, 3, and 7 (Fig. 6; Fig. 7, B and C).

It is conceivable that the positions of wt-TSHR with the described CAM influence close interaction between ECD and TMD in the wt-TSHR. Such CAM loosen this tight interaction and may allow higher affinity binding of S37a in these TSHR mutants, which could explain the strong inhibition of CAM located in the ECL, CH, and internal agonist but not of those CAM in the seven TMHs.

Each described single CAM at the ECD/TMD interface probably changes its spatial location, emphasizing delocalization of $\mathrm{CH}$ that also leads, owing to the covalent links, to a conformational change or displacement of the internal agonist. Additionally our models suggest that residues of the internal agonist E409 and/or D410 might interact with TMH6 and TMH7, rearranging the transmembranespanning helices, especially TMH 6 and 7, and thus allowing the intracellular interaction with $\mathrm{G}_{\mathrm{s}}$ protein. Charge interaction of E409 with the highly conserved K660 (TMH7) is conceivable (Fig. 6; Fig. 7C), since a single peptide of the internal agonist FNPCKDIMGY, wherein glutamate corresponding to E409 is mutated to lysine, blocks GPHR activation (Brüser et al., 2016).

In summary and on the basis of our model-guided mutations and their effects on the function of our NAM S37a, we suggest the following course for the mechanism of the intramolecular activation within TSHR: It is initiated by binding of the hormone TSH between LRRD and the hinge region of the ectodomain. At the ECD/TMD interface, this leads to rearrangements of both the converging helix and the internal agonist. Both are embedded between the extracellular loops and mediate their conformational changes, which in turn finally 
trigger the active conformations of the transmembrane helices (cartoons Fig. 9, A and B). There is an allosteric pocket between TMHs corresponding to the orthosteric rhodopsin-like ligand pocket of many GPCRs of family A and this allows a PAM, such as agonist $\mathrm{C} 2$, to activate the TSHR (Fig. 9C). From the inhibitory effects of NAM S37a on 1) ECD swapping chimeras, 2) stepwise $\mathrm{N}$-terminal truncations, 3) distinct CAM, and 4) site-directed mutants, we conclude that S37a binds to an additional pocket at the ECD/TMD interface, most probably between the converging helix, ECL1, and the internal agonist. Thus S37a is able to block both TSH- and PAM-induced molecular activation of the TSHR exactly there (Fig. 9D).

We here provide new molecular and structural insights into the interface between the extracellular domain and the transmembrane domain that is critical for activation or inhibition of the TSHR. Our proposed new allosteric ligand
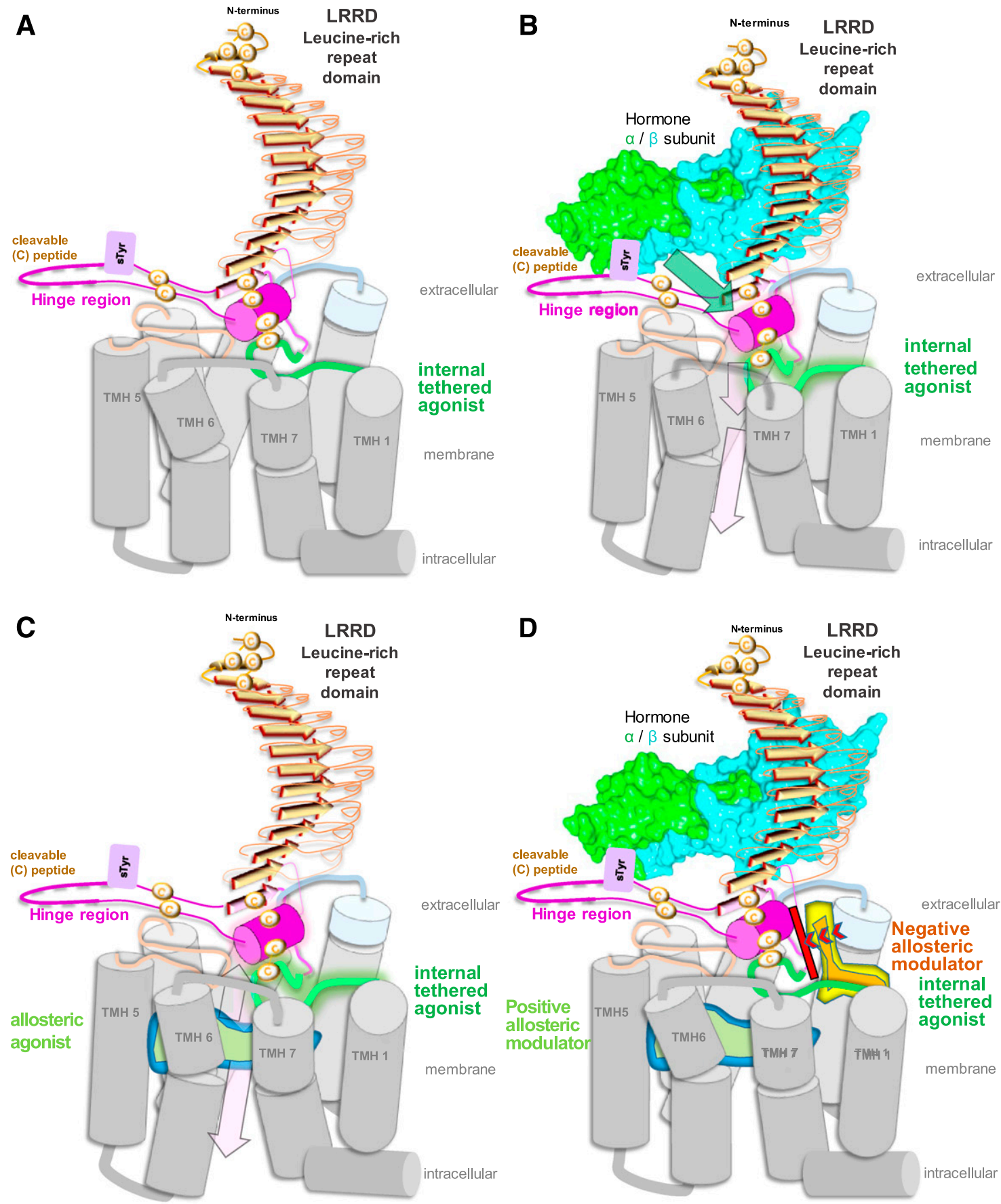

Fig. 9. TSHR cartoons for activation/inhibition signal transmission at ECD/TMD interface. (A) Unbound basal state; converging helix, CH (magenta) and inverse agonist are covalently linked by disulfide bonds. Both are immersed between the three ECL. (B) TSH bound between LRRD and sTyr385 of hinge region induce conformational changes of both $\mathrm{CH}$ and internal agonist that trigger conformational changes of ECLs and TMHs to the active state. (C) Allosteric pocket (blue) between TMHs allows small-molecule agonist (pale green) to activate the TMD. (D) An additional allosteric ligand pocket in the extracellular vestibule (yellow) between ECL1, CH and internal agonist allows our NAM S37a (orange) to freeze (red bar) $\mathrm{CH}$ and the internal agonist in an inactive conformation, which blocks the activation course of TSH and the PAM as well. 
binding pocket is located exactly at this interface and exhibits specific features that may allow the generation of potent drugs that are highly specific to TSHR and which could potentially be used for pharmacological intervention in the difficult-totreat Graves' orbitopathy (Bartalena, 2013).

\section{Acknowledgments}

We thank Jonas Protze for his help with the refinement of the images showing the S37a docking positions. We are very grateful to Edgar Specker for his support in the crystallization of S37a.

\section{Authorship Contributions}

Participated in research design: Schülein, Krause, Marcinkowski. Conducted experiments: Marcinkowski, Mendieta, Hoyer, Furkert. Contributed new reagents or analytic tools: Kreuchwig, Witte, Krause, Furkert.

Performed data analysis: Marcinkowski, Lentz, Furkert.

Wrote or contributed to the writing of the manuscript: Krause, Marcinkowski, Schülein, Lentz, Rutz.

\section{References}

Bartalena L (2013) Graves' orbitopathy: imperfect treatments for a rare disease. Eur Thyroid J 2:259-269.

Briet C, Suteau-Courant V, Munier M, and Rodien P (2018) Thyrotropin receptor, still much to be learned from the patients. Best Pract Res Clin Endocrinol Metab 32:155-164.

Brüser A, Schulz A, Rothemund S, Ricken A, Calebiro D, Kleinau G, and Schöneberg T (2016) The activation mechanism of glycoprotein hormone receptors with implications in the cause and therapy of endocrine diseases. J Biol Chem 291:508-520.

Bruysters M, Verhoef-Post M, and Themmen APN (2008) Asp330 and Tyr331 in the C-terminal cysteine-rich region of the luteinizing hormone receptor are key residues in hormone-induced receptor activation. $J$ Biol Chem 283.25821-25828.

Bruker AXS Inc. (2016) DOC-M86-EXX229 APEX3 Software User Manual, SpringerVerlag, Berlin, Heidelberg.

Farrugia LJ (1997) ORTEP -3 for Windows - a version of ORTEP -III with a Graphical User Interface (GUI). $J$ Appl Crystallogr 30:565-565.

Farrugia LJ (2012) WinGX and ORTEP for Windows : an update. J Appl Crystallogr 45:849-854.

Ho SC, Goh SS, Li S, Khoo DH, and Paterson M (2008) Effects of mutations involving cysteine residues distal to the $\mathrm{S} 281 \mathrm{HCC}$ motif at the $\mathrm{C}$-terminus on the functional characteristics of a truncated ectodomain-only thyrotropin receptor anchored on glycosylphosphatidyl-inositol. Thyroid 18:1313-1319.

Ho SC, Van Sande J, Lefort A, Vassart G, and Costagliola S (2001) Effects of mutations involving the highly conserved S281HCC motif in the extracellular domain of the thyrotropin (TSH) receptor on TSH binding and constitutive activity. Endocrinology 142:2760-2767.

Ho SN, Hunt HD, Horton RM, Pullen JK, and Pease LR (1989) Site-directed mutagenesis by overlap extension using the polymerase chain reaction. Gene 77:51-59.

Hoyer I (2014) Struktur - Funktionsanalysen intramolekularer Signalisierungsmechanismen und pharmakologische Intervention am Thyreoidea stimulierenden Hormon Rezeptor. Doctoral dissertation, Freie Universität Berlin, Berlin, Germany

Hoyer I, Haas A-K, Kreuchwig A, Schülein R, and Krause G (2013) Molecular sampling of the allosteric binding pocket of the TSH receptor provides discriminative pharmacophores for antagonist and agonists. Biochem Soc Trans 41:213-217.

Jaeschke H, Neumann S, Kleinau G, Mueller S, Claus M, Krause G, and Paschke R (2006) An aromatic environment in the vicinity of serine 281 is a structural requirement for thyrotropin receptor function. Endocrinology 147:1753-1760.

Jiang X, Liu H, Chen X, Chen P-H, Fischer D, Sriraman V, Yu HN, Arkinstall S, and $\mathrm{He} \mathrm{X}$ (2012) Structure of follicle-stimulating hormone in complex with the entire ectodomain of its receptor. Proc Natl Acad Sci USA 109:12491-12496.

Kero J, Ahmed K, Wettschureck N, Tunaru S, Wintermantel T, Greiner E, Schütz G and Offermanns S (2007) Thyrocyte-specific Gq/G11 deficiency impairs thyroid function and prevents goiter development. J Clin Invest 117:2399-2407.

Kleinau G, Haas A-K, Neumann S, Worth CL, Hoyer I, Furkert J, Rutz C, Gershengorn MC, Schülein R, and Krause G (2010) Signaling-sensitive amino acids surround the allosteric ligand binding site of the thyrotropin receptor. FASEB $J$ 24:2347-2354.

Kleinau G, Jaeschke H, Mueller S, Raaka BM, Neumann S, Paschke R, and Krause G (2008) Evidence for cooperative signal triggering at the extracellular loops of the TSH receptor. FASEB $J$ 22:2798-2808.

Kleinau G, Mueller S, Jaeschke H, Grzesik P, Neumann S, Diehl A, Paschke R, and Krause G (2011) Defining structural and functional dimensions of the extracellular thyrotropin receptor region. J Biol Chem 286:22622-22631.

Kleinau G and Vassart G (2017) TSH Receptor Mutations and Diseases, MDText.com, Inc., South Dartmouth, MA.

Kleinau G, Worth CL, Kreuchwig A, Biebermann H, Marcinkowski P, Scheerer P, and Krause G (2017) Structural-functional features of the thyrotropin receptor: a class A G-protein-coupled receptor at work. Front Endocrinol (Lausanne) 8:86.

Krause G and Marcinkowski P (2018) Intervention strategies into glycoprotein hormone receptors for modulating (Mal-)function, with special emphasis on the TSH receptor. Horm Metab Res 50:894-907.
Krause G, Kreuchwig A, and Kleinau G (2012) Extended and structurally supported insights into extracellular hormone binding, signal transduction and organization of the thyrotropin receptor. PLoS ONE 7 (12):e52920, doi: 10.1371/journal.pone.0052920 23300822 .

Kreuchwig A, Kleinau G, and Krause G (2013) Research resource: novel structural insights bridge gaps in glycoprotein hormone receptor analyses. Mol Endocrinol 27:1357-1363.

Laurent E, Mockel J, Van Sande J, Graff I, and Dumont JE (1987) Dual activation by thyrotropin of the phospholipase C and cyclic AMP cascades in human thyroid. Mol Cell Endocrinol 52:273-278.

Marcinkowski P, Hoyer I, Specker E, Furkert J, Rutz C, Neuenschwander M, Sobottka S, Sun H, Nazare M, Berchner-Pfannschmidt U, et al. (2019) A new highly thyrotropin receptor-selective small-molecule antagonist with potential for the treatment of Graves' orbitopathy. Thyroid 29:111-123.

Mueller S, Kleinau G, Jaeschke H, Neumann S, Krause G, and Paschke R (2006 Significance of ectodomain cysteine boxes 2 and 3 for the activation mechanism of the thyroid-stimulating hormone receptor. J Biol Chem 281:31638-31646.

Neumann S, Huang W, Titus S, Krause G, Kleinau G, Alberobello AT, Zheng W, Southall NT, Inglese J, Austin CP, et al. (2009) Small-molecule agonists for the thyrotropin receptor stimulate thyroid function in human thyrocytes and mice. Proc Natl Acad Sci USA 106:12471-12476.

Neumann S, Nir EA, Eliseeva E, Huang W, Marugan J, Xiao J, Dulcey AE, and Gershengorn MC (2014) A selective TSH receptor antagonist inhibits stimulation of thyroid function in female mice. Endocrinology 155:310-314.

Neumann S, Padia U, Cullen MJ, Eliseeva E, Nir EA, Place RF, Morgan SJ, and Gershengorn MC (2016) An enantiomer of an oral small-molecule TSH receptor agonist exhibits improved pharmacologic properties. Front Endocrinol (Lausanne) 7:105. Rapoport B, Chazenbalk GD, Jaume JC, and McLachlan SM (1998) The thyrotropin (TSH) receptor: interaction with TSH and autoantibodies. Endocr Rev 19:673-716

Sanders J, Chirgadze DY, Sanders P, Baker S, Sullivan A, Bhardwaja A, Bolton J, Reeve M, Nakatake N, Evans M, et al. (2007) Crystal structure of the TSH receptor in complex with a thyroid-stimulating autoantibody. Thyroid 17:395-410.

Sanders P, Young S, Sanders J, Kabelis K, Baker S, Sullivan A, Evans M, Clark J, Wilmot J, Hu X, et al. (2011) Crystal structure of the TSH receptor (TSHR) bound to a blocking-type TSHR autoantibody. J Mol Endocrinol 46:81-99.

Sato S, Noh JY, Sato S, Suzuki M, Yasuda S, Matsumoto M, Kunii Y, Mukasa K, Sugino K, Ito K, et al. (2015) Comparison of efficacy and adverse effects between methimazole $15 \mathrm{mg}+$ inorganic iodine $38 \mathrm{mg} /$ day and methimazole $30 \mathrm{mg} /$ day as initial therapy for Graves' disease patients with moderate to severe hyperthyroidism. Thyroid 25:43-50.

Schaarschmidt J, Huth S, Meier R, Paschke R, and Jaeschke H (2014) Influence of the hinge region and its adjacent domains on binding and signaling patterns of the thyrotropin and follitropin receptor. PLoS One 9:e111570.

Schaarschmidt J, Nagel MBM, Huth S, Jaeschke H, Moretti R, Hintze V, von Bergen M, Kalkhof S, Meiler J, and Paschke R (2016) Rearrangement of the extracellular domain/extracellular loop 1 interface is critical for thyrotropin receptor activation. J Biol Chem 291:14095-14108.

Sheldrick GM (2015a) Crystal structure refinement with SHELXL. Acta Crystallogr C Struct Chem 71:3-8.

Sheldrick GM (2015b) SHELXT - integrated space-group and crystal-structure determination. Acta Crystallogr A Found Adv 71:3-8.

Song Y, Massart C, Chico-Galdo V, Jin L, De Maertelaer V, Decoster C, Dumont JE, and Van Sande J (2010) Species specific thyroid signal transduction: conserved physiology, divergent mechanisms. Mol Cell Endocrinol 319:56-62.

Teichmann A, Gibert A, Lampe A, Grzesik P, Rutz C, Furkert J, Schmoranzer J, Krause G, Wiesner B, and Schülein R (2014) The specific monomer/dimer equilibrium of the corticotropin-releasing factor receptor type 1 is established in the endoplasmic reticulum. J Biol Chem 289:24250-24262.

van den Ent F and Löwe J (2006) RF cloning: a restriction-free method for inserting target genes into plasmids. J Biochem Biophys Methods 67:67-74

van Zundert GCP, Rodrigues JPGLM, Trellet M, Schmitz C, Kastritis PL, Karaca E Melquiond ASJ, van Dijk M, de Vries SJ, and Bonvin AMJJ (2016) The HADDOCK2.2 web server: user-friendly integrative modeling of biomolecular complexes. J Mol Biol 428:720-725.

Vassart G, Pardo L, and Costagliola S (2004) A molecular dissection of the glycoprotein hormone receptors. Trends Biochem Sci 29:119-126.

Vlaeminck-Guillem V, Ho S-C, Rodien P, Vassart G, and Costagliola S (2002) Activation of the cAMP pathway by the TSH receptor involves switching of the ectodomain from a tethered inverse agonist to an agonist. Mol Endocrinol 16:736-746. Wacker D, Stevens RC, and Roth BL (2017) How ligands illuminate GPCR molecular pharmacology. Cell 170:414-427.

Worth CL, Kreuchwig F, Tiemann JKS, Kreuchwig A, Ritschel M, Kleinau G, Hildebrand PW, and Krause G (2017) GPCR-SSFE 2.0-a fragment-based molecular modeling web tool for Class A G-protein coupled receptors. Nucleic Acids Res 45 (W1):W408-W415.

Zhang J, Yang J, Jang R, and Zhang Y (2015) GPCR-I-TASSER: a hybrid approach to $\mathrm{G}$ protein-coupled receptor structure modeling and the application to the human genome. Structure 23:1538-1549.

Zhang M, Tong KPT, Fremont V, Chen J, Narayan P, Puett D, Weintraub BD, and Szkudlinski MW (2000) The extracellular domain suppresses constitutive activity of the transmembrane domain of the human TSH receptor: implications for hormone-receptor interaction and antagonist design. Endocrinology 141:3514-3517. Zhang ML, Sugawa H, Kosugi S, and Mori T (1995) Constitutive activation of the thyrotropin receptor by deletion of a portion of the extracellular domain. Biochem Biophys Res Commun 211:205-210.

Address correspondence to: Dr. Gerd Krause, Leibniz-Forschungsinstitut für Molekulare Pharmakologie (FMP), Robert-Rössle-Str. 10, 13125 Berlin, Germany. E-mail: GKrause@fmp-berlin.de 